\title{
LONGITUD DE PRIMERA MADURACIÓN Y EPOCA DE DESOVE DEL DORADO (Brachyolatystoma flavicans) EN LA AMAZONIA PERUANA
}

\author{
M. Sc. Victor Montreuil * \\ Blga. Aurea García* \\ Blgo. Ronald Rodriguez ${ }^{*}$
}

\section{RESUMEN}

Brachyplatystoma aflavicans es una de las diez principales especies que sustentan la pesquería en la parte peruana de la región amazónica, de acuerdo con las estadísticas de los desembarques registrados en Iquitos-Perú, observándose un constante incremento de las capturas a través del tiempo (Tello et al. 1995). La presión de pesca sobre esta especie, en los últimos tiempos, ha sido de una magnitud tal que se han detectado especírnenes pequeños ( $82 \mathrm{~cm}$ de longitud total) en los desembarques. Estos hechos motivaron la ejecución del presente estudio, para determinar los aspectos más importantes de su biología reproductiva, calculando la talla de primera maduración y la correspondiente al primer desove como un medio de regulación de su explotación.

\section{ABSTRACT}

According to the statistics registered in Iquitos, Brachyplatystoma flavicanis is one of the ten main species that support the fishing in the area, having its catch increased with time (Tello et al., 1995). The pressure for the fishing of this species has been so hard that even small-sized fish have been detected at the port. This fact was what motivated this study so that the most important aspects of its reproductive biology may be determined, figuring the size of its first maturity and that corresponding to its first spawning in a way that its exploitation may be regulated. 


\section{INTRODUCCION}

El «dorado» (Brachyplatystoma flavicans) es una especie perteneciente a los grandes bagres de la Amazonía Peruana, cuya importancia es resaltada por el rol que cumple en la provisión de proteína animal para la población local y sus posibilidades de comercialización en los países vecinos, fundamentalmente Brasil y Colombia.

Conocer elementos importantes de su ciclo reproductivo, proporciona datos que contribuyen a regular su uso.

\section{MATERIAL $Y$ METODOS}

\subsection{La Especie}

Brachyplatystoma flavicans presenta el cuerno alargado y redondo, con la cabeza deprimida, ojos pequeños de posición superior y barbillas maxilares cortas que llegan hasta el nacimiento de las pectorales o más cortas. Proceso humeral pequeño y boca terminal con dientes pequeños y numerosos, dispuestos en banda sobre las mandíbulas y premaxilares. Aleta adiposa mediana, caudal ahorquillada y ventrales insertadas a la altura de la parte central de la dorsal y más largas que las pectorales. La coloración del cuerno es ceniza plateado, siendo el dorso más oscuro que el vientre.

\subsection{Ubicación Taxonómica (según Nelson J. 1994)}

$\begin{array}{ll}\text { Phyllum } & \text { : Chordata } \\ \text { Clase } & \text { : Osteichthyes } \\ \text { Sub-clase } & \text { : Actinopterygii } \\ \text { División } & \text { : Teleostei } \\ \text { Sub-división } & \text { : Euteleostei } \\ \text { Super-orden } & \text { : Ostariophysi } \\ \text { Orden } & \text { : Siluriformes } \\ \text { Familia } & \text { : Pimelodidae } \\ \text { Género } & \text { : Brachyplatystoma } \\ \text { Especie } & : \text { Bracchyplatystoma flavicans }\end{array}$




\subsection{Metodología}

El periodo de estudio estuvo comprendido entre mayo y diciembre de 1995, durante el cual se registró información biológica de los especimenes de Brachyplatystoma flavicans, «dorado», provenientes del Río Amazonas Peruano y Río Marañón, capturados por la flota pesquera especializada en la explotación de esta especie.

Los muestreos se realizaron de lunes a sábado, en las horas de mayor comercialización (06.00-11.00 am.), a medida que los ejemplares eran expuestos para la venta a los comerciantes minoristas.

Se midió la longitud total y la longitud a la horquilla $(\mathrm{cm})$ utilizando una cinta métrica de $10,0 \mathrm{mdc}$ longitud con aproximación a $0,2 \mathrm{~cm}$. La longitud total comprende la distancia entre el hocico y el extremo más distal de la aleta caudal, y la longitud a la horquilla desde la punta del hocico hasta el extremo posterior de los radios caudales medios; en ambos casos siguiendo la curvatura del cuerno del pez. El peso (g) se midió utilizando una balanza de reloj de $100 \mathrm{~kg}$ con sensibilidad a $250 \mathrm{~g}$. Los especimenes fueron pesados enteros, antes de la evisceración.

El sexo se determinó por exposición de la cavidad visceral, realizando un corte longitudinal desde la cintura escapular hasta el inicio de la aleta anal. Las gónadas ocupan el tercio inferior de la cavidad abdominal. Las gónadas se conservaron en fresco para su posterior pesaje y análisis en laboratorio, los cuales se realizaron dentro de las dos horas posteriores a su colección. El peso de la gónada se determinó usando una balanza Ohaus de $2610 \mathrm{~g}$ con aproximación a $0,1 \mathrm{~g}$.

Se calculó la proporción sexual mediante la distribución de frecuencias relativas de machos y hembras, aplicándose la prueba Chi Cuadrado $\left(\mathrm{X}^{2}\right)$, para determinar las diferencias significativas de la relación 1:1, según la fórmula:

$$
\mathrm{x}^{2}=\frac{(\mathrm{O}-\mathrm{E})^{2}}{\mathrm{E}} \quad \begin{aligned}
& \mathrm{x}^{2}=\text { Chi cuadrado } \\
& \mathrm{O}=\text { Frecuencia Observada } \\
& \mathrm{E}=\text { Frecuencia Esperada }
\end{aligned}
$$

Se elaboró una escala de madurez sexual de la especie en estudio para ambos sexos, considerando las características externas de las gónadas como: Longitud y ancho $(\mathrm{cm})$, peso (g), color, presencia de productos sexuales, irrigación sanguínea y textura; tomando como guía la escala presentada por Valderrama et al. (1988). 
Para los análisis de primera maduración y primer desove, se consideraron como individuos maduros a aquéllos comprendidos entre los estadios II a VI y como desovantes a las fases III a VI.

El índice gonadosomático se determinó usando la ecuación descrita en Tresierra.

$$
\mathrm{JGS}=\frac{\mathrm{Pg}}{\mathrm{Pp}} \times 100 \quad \begin{aligned}
& \mathrm{GS}=\text { Indice Gonadosomático } \\
& \mathrm{Pg}=\text { Peso de la Gónada } \\
& \mathrm{Pp}=\text { Peso del Pez }
\end{aligned}
$$

La longitud a la primera maduración se determinó agrupando los individuos maduros por sexos y por clase de longitud, estimándose las frecuencias acumuladas porcentuales para cada intervalo de longitud, y se ploteó las frecuencias acumuladas porcentuales contra los intervalos de longitud (Guerra 1980, García et al. 1995). De la curva resultante se consideró como talla de primera maduración la correspondiente a la intercepción de la curva en la frecuencia de $50 \%$.

La determinación de la época de reproducción fue establecida mediante el análisis de la abundancia mensual de especímenes maduros, muestreados durante el tiempo de estudio y la variación temporal del índice gonadosomático.

Se efectuaron análisis de regresión y correlación entre el número de especimenes maduros y algunos factores ambientales como temperatura, precipitación y niveles de río, con la finalidad de determinar la influencia medioambiental sobre el comportamiento reproductivo de Brachyplatystoma flavicans.

\section{RESULTADOS}

\subsection{Proporción por sexo}

En la Tabla 1 se presenta la composición por sexo de los ejemplares muestreados durante el período de estudio. La prueba Chi Cuadrado no encontró proporcionalidad de sexos (hembras $=56,4 \%$ o; machos $=43,6 \%$ ), siendo ellos significativamente diferentes $(\mathrm{p}=0,05)$. 
Tabla 1. Proporción de sexos y prueba Chi cuadrado para el período de estudio

\begin{tabular}{|c|c|c|c|c|}
\hline \multicolumn{2}{|c|}{$\mathrm{N}^{\circ}$ Ejemplares } & \multicolumn{2}{|c|}{ Proporción } & \multirow{2}{*}{$X_{c} 1 Q: 1 \sigma^{\prime \prime}$} \\
\hline Q & $\sigma^{\prime \prime}$ & Q & $\sigma^{*}$ & \\
\hline 225 & 174 & 1,29 & 1,00 & 6,5187 \\
\hline
\end{tabular}

$\mathrm{X}_{\mathrm{t}=3,84(\mathrm{p}=0,05)}^{2}$

\subsection{Escala de maduración}

Sobre la base de los factores indicados en la metodología se estableció una escala de maduración para Brachyplatystoma flavicans, con seis estadios que se describen en la Tabla 2.

\subsection{Tallas de primera maduración y desove}

Los machos muestran una mayor precocidad de maduración, alcanzando su primera madurez a los $107 \mathrm{~cm}$ de longitud total, mientras que las hembras lo hacen a los $127,5 \mathrm{~cm}$. (Fig. 1 y 2). Sin embargo, el desove se presenta un poco más tarde, cuando los especimenes tienen $111,3 \mathrm{~cm}$ (machos) y 130,1 cm (hembras) de longitud total (Fíg. 3 y 4 ). 
Figura 1. Talla de primera maduración de Brachyplatystoma flavicans. Hembras. Longitud total (cm)

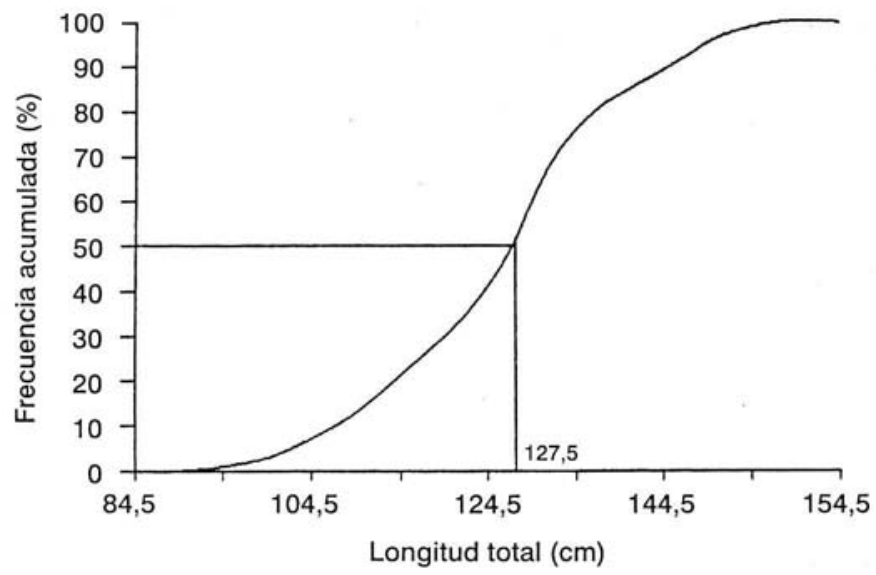

Figura 2. Talla de primera maduración de Brachyplatystoma flavicans. Machos. Longitud total (cm).

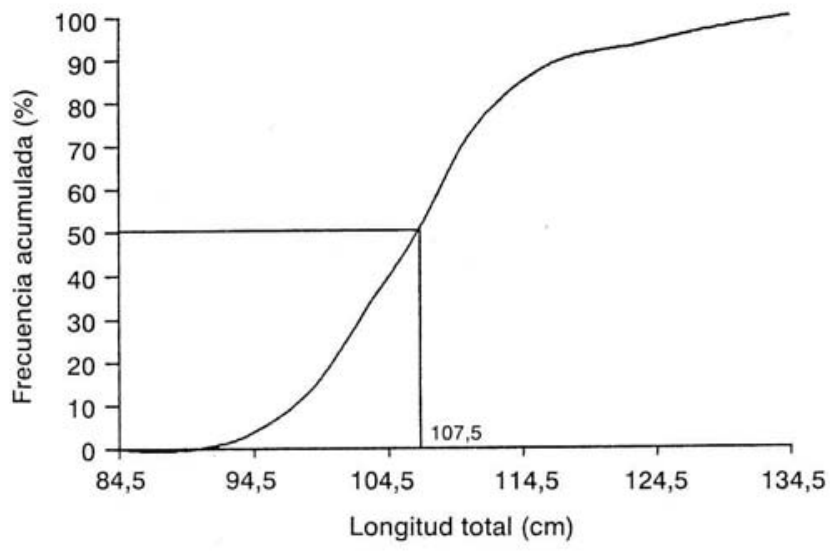


Figura 3. Talla al primer desove de Brachyplatystoma flavicans. Hembras. Longitud total (cm)

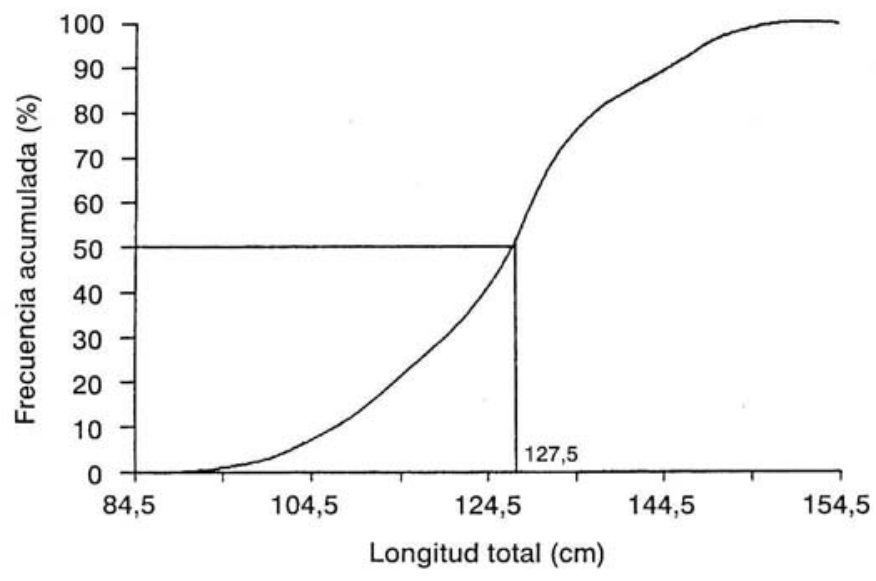

Figura 4. Talla al primer desove de Brachyplatystoma flavicans. Machos. Longitud total (cm)

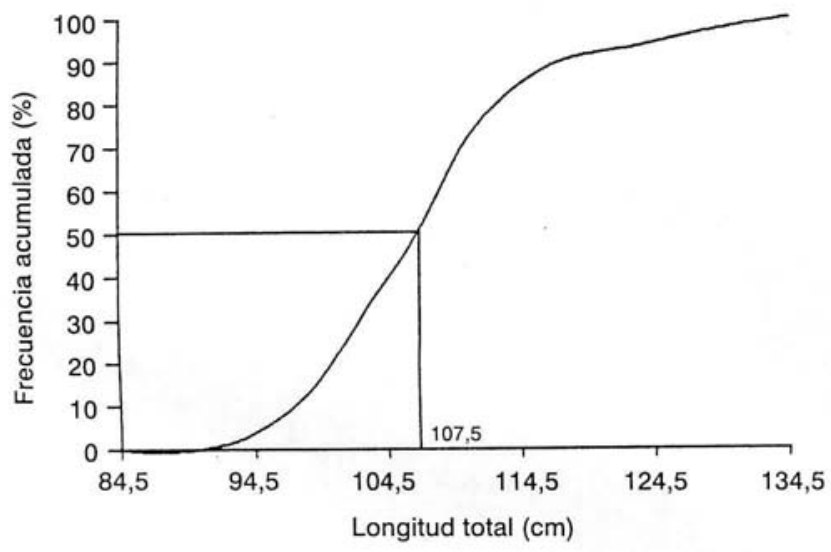


Tabla 2. Escala de maduración sexual para Brachyplatystoma Flavicans

\begin{tabular}{|c|c|}
\hline Estadio & Características de las Gónadas \\
\hline $\begin{array}{c} \\
\text { (Virgen) }\end{array}$ & $\begin{array}{l}\text { Organos sexuales pequeños. Testículos delgados y translúcidos. } \\
\text { Ovarios transparentes o de color rosado pálido, óvulos no visibles a } \\
\text { simple vista. }\end{array}$ \\
\hline $\begin{array}{c}\text { II } \\
\text { (Desarrollo) }\end{array}$ & $\begin{array}{l}\text { Testículos con filamentos bien definidos de color blanco opaco, irrigación } \\
\text { sanguínea leve en toda la gónada. Ovarios de color amarillento, óvulos } \\
\text { pequeños visibles a simple vista. }\end{array}$ \\
\hline $\begin{array}{c}\text { III } \\
\text { (Desarrollo) }\end{array}$ & $\begin{array}{l}\text { Testículos de color blanco lechoso con irrigación sanguínea bien perceptible; } \\
\text { sale esperma al presionar fuertemente la gónada. Ovarios de color amarillo, } \\
\text { irrigación sanguínea bien acentuada, óvulos grandes definidos. }\end{array}$ \\
\hline $\begin{array}{c}\text { IV } \\
\text { (En desove) }\end{array}$ & $\begin{array}{l}\text { Testículos totalmente blancos, sale esperma con leve presión. Ovarios } \\
\text { ocupan toda la cavidad abdominal, óvulos de color amarillo completamente } \\
\text { redondos y grandes. }\end{array}$ \\
\hline $\begin{array}{c}\mathrm{V} \\
\text { (Desovado) }\end{array}$ & Organos sexuales flácidos. Ovarios sanguinolentos. \\
\hline $\begin{array}{c}\mathrm{VI} \\
\text { (En reposo) }\end{array}$ & $\begin{array}{l}\text { Testículos y ovarios más desarrollados que el estadio } 1 . \\
\text { Testículos blanco rojizos y ovarios de color rojo amarillento. } \\
\text { Óvulos no visibles. }\end{array}$ \\
\hline
\end{tabular}

\subsection{Epoca de reproducción}

De acuerdo con la Fig. 5 una alta frecuencia de especimenes inmaduros (Grado 1) fueron capturados durante el período de estudio. La misma distribución mensual por grado de maduración de los especimenes muestreados y la Fig. 6 muestran la existencia de dos modos de maduración sexual, uno dc junio a agosto y otro en noviembre, siendo el primero el mayor pico de reproducción. 
Figura 5. Distribución mensual del grado de maduración de Brachyplatystoma flavicans.

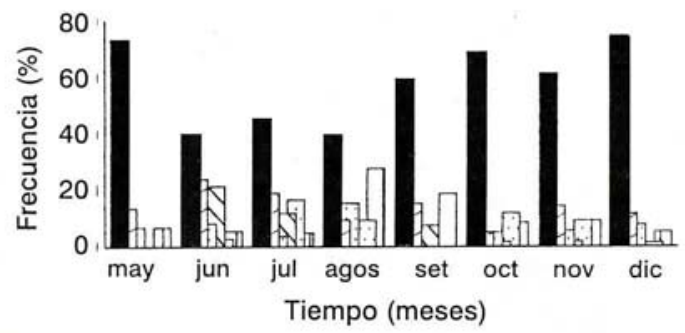

\begin{tabular}{|c|c|c|c|c|c|c|c|c|}
\hline Grado 1 & 73,3 & 39,5 & 45,3 & 39,3 & 59,3 & 68,9 & 61,4 & 74,7 \\
\hline Grado $2 \square$ & 13,3 & 23,7 & 18,6 & 9,1 & 14,8 & 4,9 & 14 & 11,4 \\
\hline Grado 3 & 6,7 & 7,9 & 3,5 & 15,2 & & 4,9 & 5,3 & 7,6 \\
\hline Grado $4 D$ & & 21,1 & 11,6 & & 7,4 & 1,6 & 1,7 & \\
\hline Grado $5 \square$ & & 2,6 & 16,3 & 9,1 & & 11,5 & 8,8 & 1.2 \\
\hline Grado 6 (1) & 6,7 & 5,2 & 4,7 & 27,3 & 18,5 & 8,2 & 8,8 & 5,1 \\
\hline
\end{tabular}

Figura 6. Distribución mensual de especimenes de maduración de Brachyplatystoma flavicans.

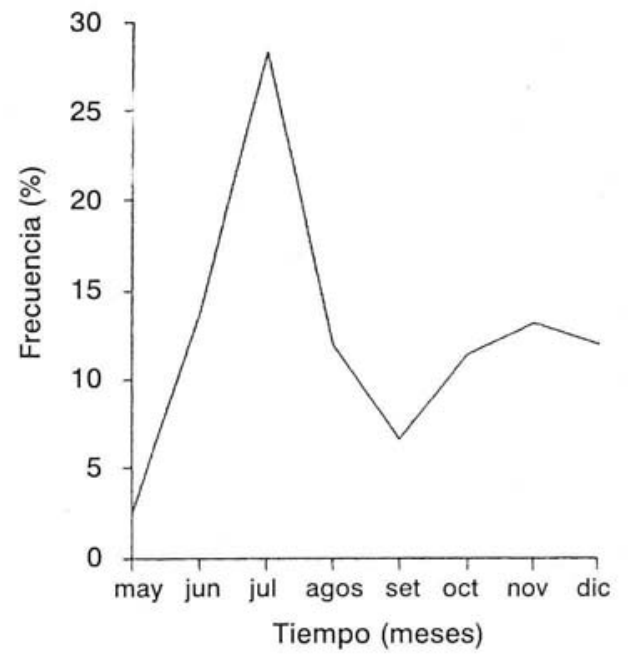


Figura 7. Distribución de especimenes de maduración de Brachyplatystoma flavicans en relación a variaciones del nivel del agua.

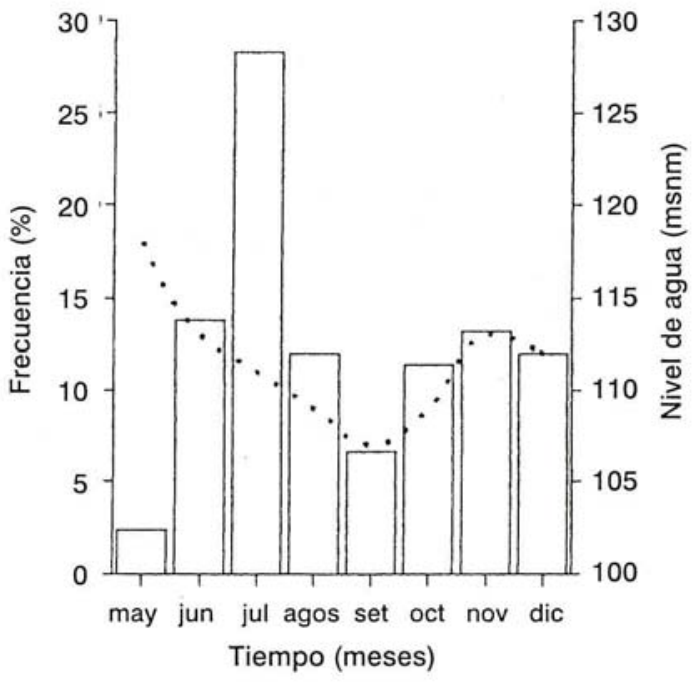

Figura 8. Distribución mensual de maduración e IGS Brachyplatystoma flavicans.

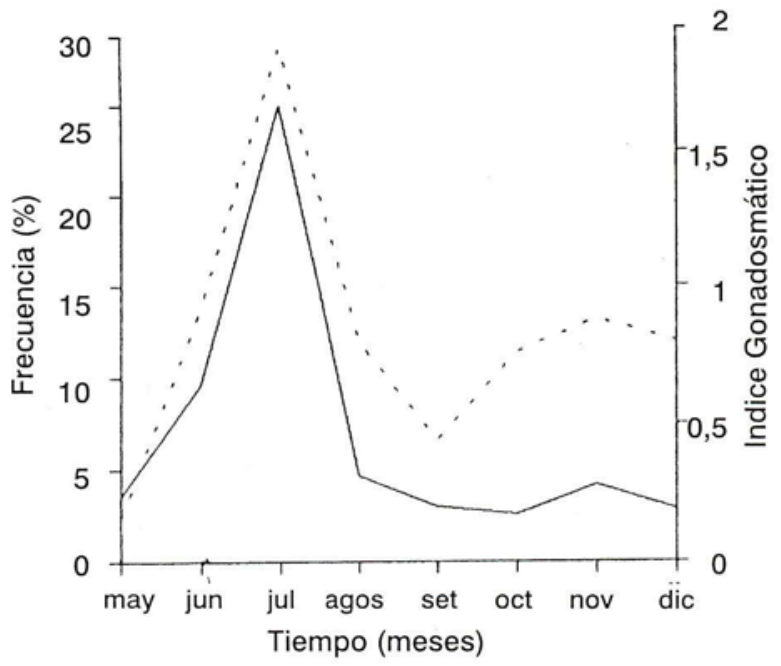


Estos picos de maduración sexual coinciden con el periodo de aguas bajas del río Amazonas (Fig. 7), sin incluir el mínimo nivel de agua que se presenta como un mecanismo de separación de los picos reproductivos mencionados.

Los períodos reproductivos de la especie son confirmados por las variaciones mensuales del Î́ndice Gonadosomático (Fig. 8), que siguen la misma tendencia de la distribución de maduración sexual (Fig. 6).

\section{DISCUSION}

La regulación de una pesquería debe asegurar que, por lo menos, el $50 \%$ de los especímenes capturados haya podido reproducirse (Valderrama et al. 1988). Considerando la información presentada en este reporte, la longitud media de captura para hembras $(118,98 \mathrm{~cm})$ y machos $(103,99 \mathrm{~cm})$ está por debajo de las tallas de primera maduración (107,0 cm y 127,5 cm, para machos y hembras respectivamente). Esto nos indica que se podría estar afectando la capacidad biogénica del Brachyplatystoma flavicans, especie caracterizada por tener un ciclo vital largo, esto es nivel trófico alto, crecimiento lento y alta fecundidad.

Aunque Valderrama (1988) afirma que $<$ el criterio ideal es mantener una talla media de captura igual a la talla media de madurez...», consideramos que para el caso de la Amazonía Peruana la talla media de madurez debe ser reemplazada por la talla media de desove, teniendo en cuenta la diferencia de aproximadamente $5 \mathrm{~cm}$ entre ambas longitudes. De esta forma aseguraríamos que el $50 \%$ del stock explotado haya desovado por lo menos una vez.

Un punto adicional a considerar es la diferencia de tallas entre los especímenes machos y hembras maduros. La separación de $20,5 \mathrm{~cm}$ entre la longitud de primera maduración entre machos $(107,0 \mathrm{~cm})$ y hembras $(127,5 \mathrm{~cm})$, crea un mayor riesgo para la reproducción de las hembras. Aunque se encuentran especimenes maduros a lo largo de todo el año, característica de muchas especies tropicales (Moreno et al. 1993), los dos picos de reproducción determinados coinciden con la época de aguas bajas, exceptuando el mínimo nivel, que asume el punto de separación de las modas reproductivas, no existiendo correlaciones significativas entre la maduración y las variaciones del nivel del río, temperatura y precipitación. 
En comparación a la talla de primera maduración de la misma especie, encontrada por Rodríguez (1991) en el Bajo Río Caquetá $-88 \mathrm{~cm}$ para hembras y $79 \mathrm{~cm}$ para machos-, y Salinas (1994) en el sector colombiano del Río Amazonas -107 cm para hembras y $104 \mathrm{~cm}$ para machos-, existen diferencias marcadas provocadas no sólo por la diferente longitud utilizada (longitud estándar), sino, consideramos, por la historia más antigua de la pesquería de dorado en las cuencas colombianas.

Tello et al. (1995), confirman el incremento de las capturas de grandes bagres desde 400 toneladas (1983) a 1500 toneladas (1994), propiciada por la alta de manda que la carne de estas especies tienen en los mercados de Colombia y Brasil. Nuestros resultados coinciden con su preocupación de que se estén capturan do especímenes inmaduros con longitudes inferiores a la talla de primera maduración.

Las conclusiones del estudio nos permiten afirmar que es necesario implementar inmediatamente medidas de regulación de la pesquería de esta especie, posibilitando que los individuos mayores a $131 \mathrm{~cm}$ (se adopta la talla de desove de las hembras debido a la utilización de métodos no selectivos para capturar esta especie) desoven por lo menos una vez en su vida y asegurando la existencia de un stock inalterado si se les somete a una explotación racional (Samamé 1992), pues ella podría colapsar en el corto plazo si no se establecen tallas mínimas de captura y períodos de veda temporal. Un argumento que se podría utilizar en contra de esta urgencia es la alta fecundidad de la especie, que propicia una rápida renovación de los stocks; sin embargo, es conveniente considerar la elevada mortalidad natural y la explotación de los juveniles de esta especie para comercializarlos corno ornamentales (Tello et al. 1995).

\section{BIBLIOGRAFIA}

GARCIA V., A.; RODRIGUEZ y., R.; GUERRA F., H. y 5. TELLO M. 1995. Madurez sexual de boquichico Prochilodus nigricans. IIAP.

GUERRA F., H. 1980. Desarrollo sexual del paiche (Arapaima gigas) en las Zonas Reservadas del Estado (Ríos Pacavay Samiria,). 19 71-1975. IMARPE Informe No 67.

MORENO T., C.; VALDERRAMA B., M., y I. BELTRÁN G. 1993. Epocas de re producción, talla media de madurez gonadal y análisis de la problemática con referencia a las tallas de raptora del bagre rayado (Pseudoplatystoma fasciatum, Linnaeus 1766) en el medio Magdalena-Sector Barrancabermeja Instituto Nacional de Pesca y Acuicultura (INPA), Colombia. 
NELSON, J. 1994. Fishes of the world. II edition. Nueva York (United States): Jc Wiley \& Sons, INC. 600 pp.

RODRIGUEZ F., C. 1991. Bagres, malleros y cuerderos en el Bajo Río Caquetá Tropenbos-Colornbia.

SALINAS C., Y. 1994. Aspectos de la biología pesquera de las poblaciones de grandes bagres (Ostariophysi: Siluriformes, Pimelodidae) en el sector colombiano del río Amazonas. Universidad Pedagógica Nacional. Facultad Ciencia y Tecnología. Colombia.

SAMAME, M. 1992. Algunos aspectos de la biología y pesquería del bonito Sarda chilensis de la costa norte del Perú. Lima (Perú): Memoria X CONABIC 02-07 agosto. pp. 26 1-272.

TELLO M., 5.; GUERRA F., H.: RODRIGUEZ V., R. y A. CARCIA V. 1995. pesquería de grandes bagres en la Región Loreto, con énfasis en dorado (Brachyplatystoma flavicans). Iquitos: JJAP.

VALDERRAMA B., M.; ZARATE V., M.; VERA V., G.; MORENO Z., CARABALLO G., P. y J. MARTINEZ R. 1988. Determinación de la tv media de madurez y análisis de la problemática con referencia a las tal medias de captura del bagre rayado (Pseudoplatystoma fasciatum) Linnaeus 1766 (Pisces: Pimelodidae) en la cuenca del río Magdalena, Colomk TRIANEA (Act. Cient. Tec. INDERENA) 2:537-550. 